\title{
Synthesis of Substituted Azetidinones Derived from Dimer of Apremilast
}

\begin{abstract}
YELLANKI JAGANNADHAM $^{1,2}$, DODDA VIVEKANANDA REDDY ${ }^{2}$, BHOOMIREDDY RAMADEVI $^{2}$ and BETHANAMUDI PRASANNA ${ }^{* 1,2}$
\end{abstract}

\author{
${ }^{* 1}$ Research Center, Department of Chemistry, Chaitanya Post Graduate College \\ (Autonomous), Kishanpura, Hanamkonda, Warangal Telangana State-506 001, India \\ ${ }^{2}$ Department of Chemistry, Jawaharlal Nehru Technological University, Hyderabad, \\ Telanagan State-500085, India \\ prasschem@gmail.com
}

Received 9 January 2019 / Accepted 5 February 2019

\begin{abstract}
Over the past decade, design of 2-azetidinones scaffold has attracted greater interest of synthesis because of more biological and pharmacological potencies. Specifically, sulfonamide rings and their derivatives represent a medicinally and pharmaceutically important class of heterocyclic motifs that are found as the core structural skeletons in a variety of several naturally occurring alkaloids. We effectively intended 4-(3-chloro-2-oxo-4-aryllazetidin-1-yl)-2'-(1-(3-ethoxy-4methoxyphenyl)-2-(methylsulfonyl)-ethyl)-2,4'-biisoindoline-1.1'3,3'-tetraones (6(a-f)). 4-Amino-2'(1-(3-ethoxy-4-methoxyphenyl)-2-(methylsulfonyl)ethyl)-2,4'-biisoindo line-1, 1', 3, 3'-tetraone $\mathbf{3}$ was prepared by condensation of 3-aminophthalic acid 2 with 1,3-ethoxy-4-methoxyphenyl)-2methylsutfonylethylamine $\mathbf{1}$ by a single step. Condensation of compound $\mathbf{3}$ with various aromatic aldehydes gives substituted Schiff base of 2'-(1-(3-ethoxy-4-methoxyphenyl)-2-(methylsulfonyl)ethyl)2,4'-biisoindoline-1,1',3,3'-tetraones (5(a-f)) which upon dehydrative annulation in the presence of chloroacetylchloride in the presence of triethylamine in DCM solvent yielded 4-(3-Chloro-2-oxo-4aryllazetidin-1-yl)-2'-(1-(3-ethoxy-4-methoxyphenyl)-2-(methylsulfonyl)-ethyl)-2,4'-biisoindoline$1.1^{\prime} 3,3^{\prime}$-tetraones $\mathbf{6}(\mathbf{a}-\mathbf{f})$. All the synthesized compounds were recognized by Infra Red-Vibrational spectroscopy, Proton Nuclear Magnetic Resonance, mass spectroscopy and their C, H, N analyses.
\end{abstract}

Keywords: Biisoindoline, Azetidinones, Aminophthalic acid, Schiff base, Chloroacetyl chloride, Dehydrative annulation

\section{Introduction}

Nitrogen containing heterocycles are important class of compounds which are present in a wide variety of bioactive natural products that are used for drug extensively. Over the past decade, design of 2-azetidinones scaffold has attracted greater interest of synthetic chemist due to immense biological and pharmacological potencies. Specifically, sulfonamide rings represent a pharmaceutically important class of heterocyclic motifs. The 2-azetidinone ring 
systems has a number of broad spectrum including penicillins, cephalosporins, carbapenems, nocardicins, monobactams, clavulanic acid, sulbactam and tazobactam which have been widely used as chemotherapeutic agents to treat bacterial infections and microbial diseases ${ }^{1}$, anti-bacterial $^{2}$, anti-convulsant ${ }^{3}$, anti-microbial ${ }^{4}$, anti-tubercular ${ }^{5}$, anti-inflammatory ${ }^{6}$, antihelmintic $^{7}$, anesthetic ${ }^{8}$, anti-oxidant ${ }^{9}$, enzyme inhibitors ${ }^{10}$ and also effective on the central nervous system ${ }^{11}$. From the literature we well knew that when one biologically active molecule linked with another more active molecule generally increases the potency.

Therefore, we were thought that to synthesize most useful drugs by incorporating azetidinones in Schiff bases (3) moiety. Keeping in view of diverse activity of azetidinone nucleus and dimer of Apremilast,

\section{Experimental}

Melting points were uncorrected. Infrared spectra were recorded by using a Bruker WM$4(\mathrm{X})$ spectrometer 577 model. ${ }^{1} \mathrm{H}$ NMR $(300 \mathrm{MHz})$ and ${ }^{13} \mathrm{C}$ NMR $(75 \mathrm{MHz})$ spectra were taken on a Bruker WM-400 spectrophotometer in DMSO- $d_{6}$ with tetramethylsilane as reference. Mass spectra (ESI) were carried out on a JEOL SX-102 spectrophotometer. Elemental analysis was done on a Carlo Erba EA 1108 automatic elemental analyzer. The chemicals and solvents were commercial grade, used without further purification. Compounds are purified by column chromatography by using alumina sheets purchased from Merck.

\section{General procedure for the synthesis of Apremilast Dimer (3)}

Compound 3-aminophthalic acid $2(0.01 \mathrm{~mol})$ was dissolved in acetonitrile $(20 \mathrm{~mL})$ and added 1,3-Ethoxy-4-methoxyphenyl)-2-methylsutfonylethylamine $\mathbf{1}(0.01 \mathrm{~mol})$. To this added slowly TEA $(0.012 \mathrm{~mol})$ and acetic acid $(10 \mathrm{~mL})$ at room temperature. The reaction was heated to temperature at $79-81{ }^{\circ} \mathrm{C}$ than the progress of the reaction was monitored by TLC. After completion of the reaction, filtered the salts and the DCM layer was washed with $5 \% \mathrm{NaHCO}_{3}$ than followed by water. The DCM solvent was removed under reduced pressure added methanol ( 5 volumes) and heated to for one hour, cooled the reaction mass solid separated, filtered and dried to give the compound Apremilast dimer $\mathbf{3}$ with good yields and purity.

Yield: $85 \%$; M.P. $>300{ }^{\circ} \mathrm{C} ; 3369,3084,2936,1176,1612,1524 ;{ }^{1} \mathrm{H}$ NMR (DMSO- $d_{6}$ ) 1.42-1.46 (t, 3H, $\left.-\mathrm{CH}_{3}\right), 2.76\left(\mathrm{~s}, 3 \mathrm{H},-\mathrm{CH}_{3}\right), 3.82\left(\mathrm{~s}, 3 \mathrm{H},-\mathrm{OCH}_{3}\right), 3.75-3.85,4.34-4.46(\mathrm{~m}$, $\left.2 \mathrm{H},-\mathrm{CH}_{2}\right), 4.05-4.13\left(\mathrm{~m}, 2 \mathrm{H},-\mathrm{CH}_{2}\right), 5.81-5.86(\mathrm{~m}, 1 \mathrm{H},-\mathrm{NCH}), 6.75-6.83(\mathrm{~m}, 1 \mathrm{H}, \mathrm{Ar}-\mathrm{H})$, 7.05-7.09 (m, 2H,-Ar), 7.63-7.66 (t, 2H, Ar-H), 7.75-7.81 (t,1H, Ar-H), 7.84-7.89 (d, 1H, Ar-H), 7.93-7.95 (d, 1H, -Ar-H), 8.88-8.91 (d, 1H, Ar-H), 9.51-9.53 (s, 2H, - $\left.\mathrm{NH}_{2}\right)$; Mass $(\mathrm{m} / \mathrm{z})$ : 564(M+1), $565(\mathrm{M}+2)$. Anal Calcd for $\mathrm{C}_{28} \mathrm{H}_{25} \mathrm{~N}_{3} \mathrm{O}_{8} \mathrm{~S}$ : C, 59.67; H, 4.47; N, 7.46. Found: C, 59.62; H, 4.42; N, 7.43.

Syntheses of substituted Schiff base of 2'-(1-(3-ethoxy-4-methoxyphenyl)-2-(methyl sulfonyl) ethyl)-2,4'-biiso indoline-1, $1^{\prime}, 3,3^{\prime}$-tetraones $\mathbf{5}(\boldsymbol{a}-\boldsymbol{f})$

A mixture of 4-amino-2'-(1-(3-ethoxy-4-methoxyphenyl)-2-(methylsulfonyl)ethyl)-2,4'biisoindoline-1, 1', 3, 3'-tetraone 3 (0.01 mol), aromatic aldehydes $\mathbf{4}(\mathbf{a}-\mathbf{f})(0.01 \mathrm{~mol})$ and 2-3 drops of glacial acetic acid in methanol $(20 \mathrm{~mL})$ was refluxed for $7 \mathrm{~h}$. The solvent was removed under reduced pressure. The residue was stirred with ice cold water $(25 \mathrm{~mL})$, filtered and dried. The crude product obtained was purified by column chromatography over silica gel (mobile phase: 1:9 ratio of $\mathrm{CHCl}_{3}$ : n-hexane) to get corresponding compounds 5(a-f). 
4-Benzylideneamino)-2'-(1-(3-ethoxy-4-methoxyphenyl)-2-(methylsulfonyl)ethyl)2,4'-biiso indoline-1,1',3,3'-tetraone (5a)

Yield: $78 \%$. M.P. $250-251{ }^{\circ} \mathrm{C}$; FT-IR $\left(\mathrm{K} \mathrm{Br}, \mathrm{v}, \mathrm{cm}^{-1}\right): 1772,1547,1528 ;{ }^{1} \mathrm{H}$ NMR (DMSO$\left.d_{6}\right): \delta 1.32\left(\mathrm{t}, 3 \mathrm{H},-\mathrm{CH}_{3}\right), 2.96\left(\mathrm{~s}, 3 \mathrm{H},-\mathrm{CH}_{3}\right), 2.98\left(\mathrm{~d}, 1 \mathrm{H},-\mathrm{CH}_{2}\right), 3.75\left(\mathrm{~s}, 3 \mathrm{H},-\mathrm{CH}_{3}\right), 4.02(\mathrm{q}$, $\left.2 \mathrm{H},-\mathrm{CH}_{2}\right), 4.18\left(\mathrm{~d}, 1 \mathrm{H},-\mathrm{CH}_{2}\right), 4.34(\mathrm{t}, 1 \mathrm{H},-\mathrm{N}-\mathrm{CH}), 6.60(\mathrm{~s}, 1 \mathrm{H}, \mathrm{Ar}-\mathrm{H}), 6.98(\mathrm{~d}, 2 \mathrm{H}, \mathrm{Ar}-\mathrm{H})$, 7.14 (m, 2H, Ar-H), 7.40 (m, 2H, Ar-H) 7.45-7.60 (m, 3H, Ar-H), 7.62-7.64 (m, 2H, Ar-H), 7.80-7.84 (m, 2H, Ar-H), $8.0(\mathrm{~s}, 1 \mathrm{H},-\mathrm{N}=\mathrm{CH}) .{ }^{13} \mathrm{C}$ NMR (DMSO- $\left.d_{6}\right): \delta$ ppm: $15.4,40.2$, $48.9,54.2,62.6,67.4,110.9,119.4,120.4,120.8,122.4,122.6,126.4,126.8,128.2,129.4$, $130.9,131.2,131.9,132.4,134.2,134.6,135.2,135.7,137.9,148.4,150.2,152.4,162.3$, 166.8, 168.4. MS (m/z):652 (M+1)' $653(\mathrm{M}+2)$; Anal. Calcd for $\mathrm{C}_{35} \mathrm{H}_{29} \mathrm{~N}_{3} \mathrm{O}_{8} \mathrm{~S}: \mathrm{C}, 64.51 ; \mathrm{H}$, 4.49; N, 6.45; Found: C, 64.49; H, 4.45; N, 6.42\%.

2'-(1-(3-Ethoxy-4-methoxyphenyl)-2-(methylsulfonyl)ethyl)-4-(2-hydroxybenzylideneamino) -2,4'-biisoindoline-1,1',3,3'-tetraone $(5 \boldsymbol{b})$

Yield: $70 \%$. M.P. $178-180{ }^{0} \mathrm{C}$; FT-IR $\left(\mathrm{KBr}, \mathrm{v}, \mathrm{cm}^{-1}\right): 1774,1546,1588 ;{ }^{1} \mathrm{H}$ NMR (DMSO$\left.d_{6}\right): \delta$ ppm: $1.34\left(\mathrm{t}, 3 \mathrm{H},-\mathrm{CH}_{3}\right), 2.92\left(\mathrm{~s}, 3 \mathrm{H},-\mathrm{CH}_{3}\right), 2.97\left(\mathrm{~d}, 1 \mathrm{H},-\mathrm{CH}_{2}\right), 3.76\left(\mathrm{~s}, 3 \mathrm{H},-\mathrm{CH}_{3}\right)$, $4.08\left(\mathrm{q}, 2 \mathrm{H},-\mathrm{CH}_{2}\right), 4.24\left(\mathrm{~d}, 1 \mathrm{H},-\mathrm{CH}_{2}\right), 4.38(\mathrm{t}, 1 \mathrm{H},-\mathrm{N}-\mathrm{CH}), 6.64(\mathrm{~s}, 1 \mathrm{H}, \mathrm{Ar}-\mathrm{H}), 7.02(\mathrm{~d}, 2 \mathrm{H}$, Ar-H), 7.20 (m, 2H, Ar-H), 7.44 (m, 2H, Ar-H) 7.64-7.72 (m, 4H, Ar-H), 7.82-7.86 (m, 2H, Ar-H), 8.12 (s, $1 \mathrm{H},-\mathrm{N}=\mathrm{CH}) .{ }^{13} \mathrm{C}$ NMR $\left(75 \mathrm{MHz}, \mathrm{DMSO}-\mathrm{d}_{6}\right): \delta 15.2,40.5,49.7,54.4,63.7$, $69.2,110.5,119.1,120.4,121.3,122.4,123.2,123.6,124.7,128.5,129.1,129.4,131.2$, 133.1, 133.9, 134.5, 135.2, 135.4.135.9, 136.2, 137.9, 148.7, 150.5, 151.4, 160.0, 163.2, 167.8, 168.4. MS $(\mathrm{m} / \mathrm{z}): 668(\mathrm{M}+1)^{+,} 669(\mathrm{M}+2)$; Anal. Calcd. for $\mathrm{C}_{35} \mathrm{H}_{29} \mathrm{~N}_{3} \mathrm{O}_{9} \mathrm{~S}: \mathrm{C}, 62.96 ; \mathrm{H}$, 4.38; N, 6.29; Found: C, 62.92; H, 4.34; N, 6.31\%.

2'-(1-(3-Ethoxy-4-methoxyphenyl)-2-(methylsulfonyl)ethyl)-4-(3-nitrobenzylideneamino)-2, 4' - biisoindoline-1,1',3,3'-tetraone (5c)

Yield: $68 \%$. M.P. $222-223{ }^{\circ} \mathrm{C}$; FT-IR $\left(\mathrm{K} \mathrm{Br}, \mathrm{v}, \mathrm{cm}^{-1}\right)$ : $1772,1542,1590 ;{ }^{1} \mathrm{H}$ NMR (DMSO- $d_{6}$ ): $\delta$ ppm: $1.33\left(\mathrm{t}, 3 \mathrm{H},-\mathrm{CH}_{3}\right), 2.98\left(\mathrm{~s}, 3 \mathrm{H},-\mathrm{CH}_{3}\right), 3.02\left(\mathrm{~d}, 1 \mathrm{H},-\mathrm{CH}_{2}\right), 3.76\left(\mathrm{~s}, 3 \mathrm{H},-\mathrm{CH}_{3}\right), 4.12(\mathrm{q}$, $\left.2 \mathrm{H},-\mathrm{CH}_{2}\right), 4.27\left(\mathrm{~d}, 1 \mathrm{H},-\mathrm{CH}_{2}\right), 4.35(\mathrm{t}, 1 \mathrm{H},-\mathrm{N}-\mathrm{CH}), 6.69(\mathrm{~s}, 1 \mathrm{H}, \mathrm{Ar}-\mathrm{H}), 7.09-7.13$ (d, 2H, Ar$\mathrm{H})$, 7.24-7.27 (m, 2H, Ar-H), 7.44-7.49 (m, 2H, Ar-H) 7.64-7.72 (m, 4H, Ar-H), 7.74-7.46 (s, $1 \mathrm{H}, \mathrm{Ar}-\mathrm{H}), 8.22(\mathrm{~s}, 1 \mathrm{H},-\mathrm{N}=\mathrm{CH}), 8.26-8.28(\mathrm{~d}, 1 \mathrm{H}, \mathrm{Ar}-\mathrm{H}) .{ }^{13} \mathrm{C}$ NMR $\left(75 \mathrm{MHz}, \mathrm{DMSO}_{-} \mathrm{d}_{6}\right): \delta$ $14.8,41.2,48.5,53.7,62.8,67.2,110.3,119.7,120.1,120.6,122.4,122.6,126.0,126.8,127.4$, $129.4,130.2,130.9,131.2,134.2,134.6,135.2,135.7,136.8,137.9,138.9,147.2,149.9$, 150.7, 153.1, 162.9, 165.5, 167.2.MS (m/z):671 (M+1),672 (M+2); Anal. Calcd for $\mathrm{C}_{35} \mathrm{H}_{28}$ $\mathrm{N}_{4} \mathrm{O}_{10} \mathrm{~S}: \mathrm{C}, 60.34 ; \mathrm{H}, 4.05 ; \mathrm{N}, 8.04$; Found: C, 60.21; H, 3.98; N, 8.09\%.

4-(2,3-Dichlorobenzylideneamino)-2'-(1-(3-ethoxy-4-methoxyphenyl)-2-(methylsulfonyl) ethyl)-2, 4' -biisoindoline-1,1',3,3'-tetraone (5d)

Yield: $65 \%$. M.P. $263-264{ }^{\circ} \mathrm{C}$; FT-IR $\left(\mathrm{K} \mathrm{Br}, \mathrm{v}, \mathrm{cm}^{-1}\right)$ : 1764, 1549, 1592; ${ }^{1} \mathrm{H}$ NMR (DMSO$\left.d_{6}\right): \delta$ ppm: $1.36\left(\mathrm{t}, 3 \mathrm{H},-\mathrm{CH}_{3}\right), 2.95\left(\mathrm{~s}, 3 \mathrm{H},-\mathrm{CH}_{3}\right), 3.02\left(\mathrm{~d}, 1 \mathrm{H},-\mathrm{CH}_{2}\right), 3.78\left(\mathrm{~s}, 3 \mathrm{H},-\mathrm{CH}_{3}\right)$, $4.10\left(\mathrm{q}, 2 \mathrm{H},-\mathrm{CH}_{2}\right), 4.24\left(\mathrm{~d}, 1 \mathrm{H},-\mathrm{CH}_{2}\right), 4.42(\mathrm{t}, 1 \mathrm{H},-\mathrm{N}-\mathrm{CH}), 6.64(\mathrm{~s}, 1 \mathrm{H}, \mathrm{Ar}-\mathrm{H}), 6.90(\mathrm{~d}, 2 \mathrm{H}$, Ar-H), 7.16-7.18 (m, 2H, Ar-H), 7.47-7.52 (m, 2H, Ar-H) 7.62-7.64 (m, 3H, Ar-H), 7.69 $(\mathrm{s}, 1 \mathrm{H}, \mathrm{Ar}-\mathrm{H}), 8.10(\mathrm{~s}, 1 \mathrm{H},-\mathrm{N}=\mathrm{CH}), 8.21(\mathrm{~s}, 1 \mathrm{H}, \mathrm{Ar}-\mathrm{H}) .{ }^{13} \mathrm{C}$ NMR (DMSO- $\left.d_{6}\right): \delta 15.2,40.7$, $47.3,54.2,62.1,66.7,111.2,119.1,120.2,121.2,122.7,123.2,126.9,126.2,129.9,129.1$, $130.5,131.9,134.2,135.4,134.8,135.2,135.9,136.5,137.1,138.2,142.9,148.2,150.0$, 152.9, 162.5, 166.1, 167.6. MS $(\mathrm{m} / \mathrm{z}): 720(\mathrm{M}+1), 721 \quad(\mathrm{M}+2)$; Anal. Calcd. for $\mathrm{C}_{35} \mathrm{H}_{27} \mathrm{Cl}_{2} \mathrm{~N}_{3} \mathrm{O}_{8} \mathrm{~S}$ : C, 58.34; H, 3.78; N, 5.83; Found: C, 58.30; H, 3.73; N, 5.79\%. 
2'-(1-(3-Ethoxy-4-methoxyphenyl)-2-(methylsulfonyl)ethyl)-4-(4-methylbenzylideneamino)-2, 4'-biisoindoline-1,1',3,3'-tetraone $(5 \boldsymbol{e})$

Yield: $68 \%$. M.P. $244-245{ }^{\circ} \mathrm{C}$; FT-IR $\left(\mathrm{KBr}, \mathrm{v}, \mathrm{cm}^{-1}\right)$ : 1768, 1548, 1585; ${ }^{1} \mathrm{H}$ NMR (DMSO$\left.d_{6}\right): \delta$ ppm: $1.32\left(\mathrm{t}, 3 \mathrm{H},-\mathrm{CH}_{3}\right), 2.43\left(\mathrm{~s}, 3 \mathrm{H},-\mathrm{CH}_{3}\right), 2.90\left(\mathrm{~s}, 3 \mathrm{H},-\mathrm{CH}_{3}\right), 2.99\left(\mathrm{~d}, 1 \mathrm{H},-\mathrm{CH}_{2}\right)$, $3.72\left(\mathrm{~s}, 3 \mathrm{H},-\mathrm{CH}_{3}\right), 4.08\left(\mathrm{q}, 2 \mathrm{H},-\mathrm{CH}_{2}\right), 4.22\left(\mathrm{~d}, 1 \mathrm{H},-\mathrm{CH}_{2}\right), 4.36(\mathrm{t}, 1 \mathrm{H},-\mathrm{N}-\mathrm{CH}), 6.62(\mathrm{~s}, 1 \mathrm{H}$, Ar-H), 6.96-7.02 (d, 2H, Ar-H), 7.20-7.24 (m, 2H, Ar-H), 7.30-7.33 (dd, 2H, Ar-H), 7.42$7.46(\mathrm{~m}, 2 \mathrm{H}, \mathrm{Ar}-\mathrm{H}), 7.68-7.70(\mathrm{dd}, 2 \mathrm{H}, \mathrm{Ar}-\mathrm{H}), 8.12(\mathrm{~s}, 1 \mathrm{H},-\mathrm{N}=\mathrm{CH}), 8.24(\mathrm{~m}, 2 \mathrm{H}, \mathrm{Ar}-\mathrm{H})$. ${ }^{13} \mathrm{C}$ NMR (DMSO- $d_{6}$ ): $\delta$ 15.7, 23.3, 40.5, 49.3, 54.5, 62.6, 68.4, 111.3, 119.9, 121.2, $121.8,122.9,123.5,126.8,129.4,130.2,130.9,131.2,131.4,134.2,134.2,134.6,135.2$, 135.7, 137.9, 141.6, 148.4, 150.2, 152.4, 160.3, 166.8, 168.4. MS (m/z):666 (M+1), 667 $(\mathrm{M}+2)$; Anal. Calcd. for $\mathrm{C}_{36} \mathrm{H}_{31} \mathrm{~N}_{3} \mathrm{O}_{8} \mathrm{~S}$ : C, 64.95; H, 4.69; N, 6.31; Found: C, 64.92; H, $4.67 ; \mathrm{N}, 6.29 \%$.

2'-(1-(3-Ethoxy-4-methoxyphenyl)-2-(methylsulfonyl)ethyl)-4-(4-methoxybenzylideneamino) -2,4'-biisoindoline-1, 1',3,3'-tetraone (5f)

Yield: $72 \%$. M.P.184- $185{ }^{\circ} \mathrm{C}$; FT-IR $\left(\mathrm{K} \mathrm{Br}, \mathrm{v}, \mathrm{cm}^{-1}\right)$ : 1768, 1542, 1590; ${ }^{1} \mathrm{H}$ NMR (DMSO$\left.d_{6}\right): \delta$ ppm: $1.37\left(\mathrm{t}, 3 \mathrm{H},-\mathrm{CH}_{3}\right), 2.92\left(\mathrm{~s}, 3 \mathrm{H},-\mathrm{CH}_{3}\right), 3.04\left(\mathrm{~d}, 1 \mathrm{H},-\mathrm{CH}_{2}\right), 3.78\left(\mathrm{~s}, 3 \mathrm{H},-\mathrm{OCH}_{3}\right)$, $3.83\left(\mathrm{~s}, 3 \mathrm{H},-\mathrm{OCH}_{3}\right), 4.10\left(\mathrm{q}, 2 \mathrm{H},-\mathrm{CH}_{2}\right), 4.24\left(\mathrm{~d}, 1 \mathrm{H},-\mathrm{CH}_{2}\right), 4.33(\mathrm{t}, 1 \mathrm{H},-\mathrm{N}-\mathrm{CH}), 6.64(\mathrm{~s}$, 1H, Ar-H), 7.02-7.06 (d, 2H, Ar-H), 7.18-7.24 (m, 2H, Ar-H), 7.36-7.37 (dd, 2H, Ar-H), 7.50-7.54 (m, 2H, Ar-H), 7.72-7.78 (dd, 2H, Ar-H), 7.87-7.90 (m, 2H, Ar-H), 8.24 (s, 1H, $\mathrm{N}=\mathrm{CH}$ ). ${ }^{13} \mathrm{C}$ NMR (DMSO- $d_{6}$ ): $\delta 15.1,40.5,47.7,54.2,57.9,62.5,66.8,110.7,112.2$, $119.9,120.9,121.2,122.6,123.7,126.4,126.8,128.7,129.1,130.2,131.2,131.8,133.7$, 134.3, 135.7, 136.2, 138.2, 148.1, 149.7, 152.0, 161.5, 163.8, 167.2, 169.2. MS (m/z):682 $(\mathrm{M}+1), 683(\mathrm{M}+2)$; Anal Calcd for $\mathrm{C}_{36} \mathrm{H}_{31} \mathrm{~N}_{3} \mathrm{O}_{9} \mathrm{~S}: \mathrm{C}, 63.43 ; \mathrm{H}, 4.58$; N, 6.16; Found: $\mathrm{C}$, $63.41 ; \mathrm{H}, 4.56 ; \mathrm{N}, 6.14 \%$.

\section{Synthesis of Schiff bases to Azetidinones $6(\boldsymbol{a}-\boldsymbol{f})$}

Triethyl amine $(1.2 \mathrm{mmol})$ added to a stirred solution of Schiff base 5(a-f) $(1.0 \mathrm{mmol})$ in dichloromethane as solvent $(25 \mathrm{~mL})$. Chloro acetyl Chloride $(2.0 \mathrm{mmol})$ was added drop wise at $0-5{ }^{\circ} \mathrm{C}$ and the reaction mixture was stirred for overnight. The reaction mixture was poured into cooled water and the liberated compound was extracted with chloroform. Evaporation of the compound afforded the corresponding azetidinones crude. The crude product obtained was purified by column chromatography technique (mobile phase: $\mathrm{CHCl}_{3}$ / $n$-hexane 3:7).

4-(3-Chloro-2-oxo-4-phenylazetidin-1-yl)-2'-(1-(3-ethoxy-4-methoxyphenyl)-2(methylsulfo-nyl)ethyl)-2,4'-biisoindoline-1.1'3,3'-tetraone(6a)

Yield: 70\%; M.P. $243-245{ }^{\circ} \mathrm{C}$; FT-IR (K Br,v, $\left.\mathrm{cm}^{-1}\right)$ : 1774, 1738, 1590, 1052; ${ }^{1} \mathrm{H}$ NMR $\left(\right.$ DMSO- $\left.d_{6}\right): \delta 1.34\left(\mathrm{t}, 3 \mathrm{H},-\mathrm{CH}_{3}\right), 2.92\left(\mathrm{~s}, 3 \mathrm{H},-\mathrm{CH}_{3}\right), 3.78\left(\mathrm{~s}, 3 \mathrm{H},-\mathrm{CH}_{3}\right), 3.90-3.98(\mathrm{q}, 2 \mathrm{H},-$ $\left.\mathrm{CH}_{2}\right), 4.14\left(\mathrm{~d}, 1 \mathrm{H},-\mathrm{CH}_{2}\right), 4.18-4.20\left(\mathrm{~d}, 1 \mathrm{H},-\mathrm{CH}_{2}\right), 4.72(\mathrm{t}, 1 \mathrm{H},-\mathrm{N}-\mathrm{CH}), 5.38(\mathrm{~s}, 1 \mathrm{H},-\mathrm{CH})$, $5.74(\mathrm{~d}, 1 \mathrm{H},-\mathrm{CH}), 6.54(\mathrm{~d}, 1 \mathrm{H}, \mathrm{Ar}-\mathrm{H}), 6.80-6.84(\mathrm{~d}, 2 \mathrm{H}, \mathrm{Ar}-\mathrm{H}), 7.48(\mathrm{~d}, 1 \mathrm{H}, \mathrm{Ar}-\mathrm{H}), 7.28-$ $7.36(\mathrm{~m}, 5 \mathrm{H}, \mathrm{Ar}-\mathrm{H}), 7.60(\mathrm{~d}, 1 \mathrm{H}, \mathrm{Ar}-\mathrm{H}), 7.62(\mathrm{~d}, 1 \mathrm{H}, \mathrm{Ar}-\mathrm{H}), 7.83(\mathrm{t}, 1 \mathrm{H}, \operatorname{Ar}-\mathrm{H}), 7.92(\mathrm{t}, 1 \mathrm{H}$, Ar-H), $8.14(\mathrm{~d}, 1 \mathrm{H}, \mathrm{Ar}-\mathrm{H}) .{ }^{13} \mathrm{C}$ NMR (DMSO- $\left.d_{6}\right): \delta 14.2,44.2,48.8,57.2,63.4,66.4,67.2$, $69.7,110.2,120.4,120.4,120.4,121.2,122.4,125.5,126.9,128.4,128.8,130.4,134.2$, 134.2, 135.8, 136.2, 137.4, 137.2, 137.2, 138.3, 145.2, 148.9, 150.4, 160.4, 165.4, 170.2. MS (m/z):728 (M+1), $729(\mathrm{M}+2)$; Anal Calcd for $\mathrm{C}_{37} \mathrm{H}_{30} \mathrm{ClN}_{3} \mathrm{O}_{9} \mathrm{~S}: \mathrm{C}, 61.03 ; \mathrm{H}, 4.15 ; \mathrm{N}$, 5.77; Found: C, 61.01; H, 4.17; N, 5.72\%. 
4-(3-Chloro-2-(2-hydroxyphenyl)-4-oxoazetidin-1-yl)-2'-(1-(3-ethoxy-4-methoxyphenyl)-2-(methylsulfonyl)ethyl)-2,4'-biisoindoline-1.1'3,3'-tetraone(6b)

Yield: 68\%; M.P. $230-231{ }^{\circ} \mathrm{C}$; FT-IR $\left(\mathrm{KBr}, \mathrm{v}, \mathrm{cm}^{-1}\right)$ : 1772, 1738, 1590, 1052; ${ }^{1} \mathrm{H}$ NMR $\left(\mathrm{DMSO}-d_{6}\right): \delta$ ppm: $1.35\left(\mathrm{t}, 3 \mathrm{H},-\mathrm{CH}_{3}\right), 2.96\left(\mathrm{~s}, 3 \mathrm{H},-\mathrm{CH}_{3}\right), 3.74\left(\mathrm{~s}, 3 \mathrm{H},-\mathrm{CH}_{3}\right), 3.98-4.02(\mathrm{q}$, $\left.2 \mathrm{H},-\mathrm{CH}_{2}\right), 4.14\left(\mathrm{~d}, 1 \mathrm{H},-\mathrm{CH}_{2}\right), 4.22(\mathrm{~d}, 1 \mathrm{H},-\mathrm{CH}), 4.78(\mathrm{~d}, 2 \mathrm{H},-\mathrm{CH}), 5.70(\mathrm{~d}, 1 \mathrm{H},-\mathrm{CH}), 6.98$ (d, 2H, Ar-H), 7.24 (s, 1H, Ar-H), 7.68-7.73 (m, 4H, Ar-H), 7.78 (d, 1H, Ar-H), 7.90-7.98 $(\mathrm{m}, 2 \mathrm{H}, \mathrm{Ar}-\mathrm{H}), 8.02(\mathrm{~d}, 2 \mathrm{H}, \mathrm{Ar}-\mathrm{H}), 8.60(\mathrm{~d}, 1 \mathrm{H}, \mathrm{Ar}-\mathrm{H}) .{ }^{13} \mathrm{C}$ NMR (DMSO- $\left.d_{6}\right): \delta 14.4,44.8$, 48.7, 57.5, 63.9, 66.7, 67.5, 69.5, 109.7, 116.4, 120.0, 121.2, 121.9, 122.5, 123.6, 125.7, $126.2,127.7,128.3,131.5,134.2,135.1,135.4,136.9,137.5,138.2,139.5,140.3,148.9$, 150.2, 155.8, 160.2, 164.7, 171.4. MS $(\mathrm{m} / \mathrm{z}): 744(\mathrm{M}+1), 745(\mathrm{M}+2)$; Anal Calcd for $\mathrm{C}_{37} \mathrm{H}_{30}$ $\mathrm{ClN}_{3} \mathrm{O}_{10} \mathrm{~S}: \mathrm{C}, 59.72 ; \mathrm{H}, 4.06$; N, 5.65; Found: C, 59.70; H, 4.02; N, 5.61\%.

4-(3-Chloro-2-(3-nitrophenyl)-4-oxoazetidin-1-yl)-2'-(1-(3-ethoxy-4-methoxyphenyl)2-(methylsulfonyl)ethyl)-2,4'-biisoindoline-1.1'3,3'-tetraone $(\mathbf{6} \boldsymbol{c})$

Yield: $62 \%$; M.P. $263-264{ }^{\circ} \mathrm{C}$; FT-IR $\left(\mathrm{K} \mathrm{Br}, \nu, \mathrm{cm}^{-1}\right)$ : 1768, 1735, 1592, 1052; ${ }^{1} \mathrm{H}$ NMR $\left(\mathrm{DMSO}-d_{6}\right): \delta 1.36\left(\mathrm{t}, 3 \mathrm{H},-\mathrm{CH}_{3}\right), 2.90\left(\mathrm{~s}, 3 \mathrm{H},-\mathrm{CH}_{3}\right), 3.72\left(\mathrm{~s}, 3 \mathrm{H},-\mathrm{OCH}_{3}\right), 3.98(\mathrm{~d}, 1 \mathrm{H},-$ $\left.\mathrm{CH}_{2}\right), 4.07\left(\mathrm{q}, 2 \mathrm{H},-\mathrm{CH}_{2}\right), 4.12\left(\mathrm{~d}, 1 \mathrm{H},-\mathrm{CH}_{2}\right), 4.32(\mathrm{t}, 1 \mathrm{H},-\mathrm{N}-\mathrm{CH}), 5.62(\mathrm{~d}, 1 \mathrm{H},-\mathrm{CH}), 5.72$ (d, 1H, -CH), 6.92-6.95 (d, 2H, Ar-H), 7.52- 7.55 (m, 2H, Ar-H), 7.60-7.66 (m, 2H, Ar-H), 7.74-7.78 (m, 2H, Ar-H), $7.90(\mathrm{~m}, 1 \mathrm{H}, \mathrm{Ar}-\mathrm{H}), 7.94(\mathrm{~m}, 1 \mathrm{H}, \mathrm{Ar}-\mathrm{H}), 8.12-8.22(\mathrm{~m}, 2 \mathrm{H}, \mathrm{Ar}-\mathrm{H})$, $8.34(\mathrm{~d}, 1 \mathrm{H}, \mathrm{Ar}-\mathrm{H}) .{ }^{13} \mathrm{C}$ NMR $\left(\mathrm{DMSO}-d_{6}\right) \delta \mathrm{ppm}: 14.1,44.2,48.5,57.0,63.9,65.9,67.5$, $69.7,110.3,119.7,120.2,120.9,121.8,122.5,123.2,124.7,125.4,127.4,129.9,131.7$, 133.8, 134.2, 134.9, 136.2, 137.5, 137.9, 138.0, 138.7, 144.6, 147.8, 149.2, 159.2, 164.2, 169.7. MS (m/z):773 (M+1), $774(\mathrm{M}+2)$; Anal. Calcd. for $\mathrm{C}_{37} \mathrm{H}_{29} \mathrm{ClN}_{4} \mathrm{O}_{11} \mathrm{~S}: \mathrm{C}, 57.48 ; \mathrm{H}$, 3.78 ; N, 7.25; Found: C, 59.45; H, 3.73; N, 7.21\%.

4-(3-Chloro-2-(2,3-dichlorophenyl)-4-oxoazetidin-1-yl)-2'-(1-(3-ethoxy-4-methoxyphenyl)-2-(methylsulfonyl)ethyl)-2,4'-biisoindoline-1.1'3,3'-tetraone(6d)

Yield: 58\%; M.P. $221-222{ }^{\circ} \mathrm{C}$; FT-IR $\left(\mathrm{K} \mathrm{Br}, \mathrm{v}, \mathrm{cm}^{-1}\right)$ : 1776, 1742, 1589, 1052; ${ }^{1} \mathrm{H}$ NMR (DMSO$\left.d_{6}\right) \delta$ ppm: $1.35\left(\mathrm{t}, 3 \mathrm{H},-\mathrm{CH}_{3}\right), 2.89\left(\mathrm{~s}, 3 \mathrm{H},-\mathrm{CH}_{3}\right), 3.35(\mathrm{t}, 1 \mathrm{H},-\mathrm{CH}), 3.85\left(\mathrm{~s}, 3 \mathrm{H},-\mathrm{OCH}_{3}\right), 3.98-$ $4.02\left(\mathrm{q}, 2 \mathrm{H},-\mathrm{CH}_{2}\right), 4.12\left(\mathrm{~d}, 1 \mathrm{H},-\mathrm{CH}_{2}\right), 4.15(\mathrm{t}, 1 \mathrm{H},-\mathrm{N}-\mathrm{CH}), 5.24(\mathrm{~d}, 1 \mathrm{H},-\mathrm{CH}), 5.70(\mathrm{~d}, 1 \mathrm{H},-\mathrm{CH})$, 6.92-6.97 (d, 2H, Ar-H), 7.02-709 (d, 2H, Ar-H), 7.52-7.54 (t, 1H, Ar-H), 7.78-7.82 (m, 2H, Ar$\mathrm{H})$, 7.85-7.92 (m, 1H, Ar-H), 8.04-8.06 (d, 2H, Ar-H), 8.60 (d, 1H, Ar-H). ${ }^{13} \mathrm{C}$ NMR (DMSO-d $\left.{ }_{6}\right)$ $\delta$ ppm: 14.2, 44.7, 48.4, 57.2, 61.4, 62.7, 67.2, 68.9, 110.5, 119.8, 120.8, 121.2, 121.9, 123.2, $124.4,125.7,126.5,127.5,128.2,129.7,133.7,134.5,135.2,136.7,137.2,138.4,138.2,139.5$, 144.2, 147.5, 149.7, 160.0, 163.2, 169.8. MS (m/z):796 (M+1), $797(\mathrm{M}+2)$; Anal. Calcd. for $\mathrm{C}_{37} \mathrm{H}_{28} \mathrm{Cl}_{3} \mathrm{~N}_{3} \mathrm{O}_{9} \mathrm{~S}$ : C, 55.75; H, 3.54; N, 5.27; Found: C, 57.73; H, 3.51; N, 5.24\%.

4-(3-Chloro-2-oxo-4-p-tolylazetidin-1-yl)-2'-(1-(3-ethoxy-4-methoxyphenyl)-2(methylsulfon -yl) ethyl)-2,4'-biisoindoline-1.1'3,3'-tetraone $(6 \mathrm{e})$

Yield: 68\%; M.P. $277-278{ }^{\circ} \mathrm{C}$ FT-IR $\left(\mathrm{K} \mathrm{Br}, \nu, \mathrm{cm}^{-1}\right)$ : 1768, 1737, 1592, 1054; ${ }^{1} \mathrm{H}$ NMR (DMSO$\left.d_{6}\right): \delta$ ppm $1.34\left(\mathrm{t}, 3 \mathrm{H},-\mathrm{CH}_{3}\right), 2.23\left(\mathrm{~s}, 3 \mathrm{H},-\mathrm{CH}_{3}\right), 2.90\left(\mathrm{~s}, 3 \mathrm{H},-\mathrm{CH}_{3}\right), 3.36(\mathrm{t}, 1 \mathrm{H},-\mathrm{CH}), 3.82(\mathrm{~s}$, $\left.3 \mathrm{H},-\mathrm{OCH}_{3}\right), 4.02-4.06\left(\mathrm{q}, 2 \mathrm{H},-\mathrm{CH}_{2}\right), 4.12\left(\mathrm{t}, 1 \mathrm{H},-\mathrm{CH}_{2}\right), 4.62(\mathrm{t}, 1 \mathrm{H},-\mathrm{N}-\mathrm{CH}), 5.29(\mathrm{~d}, 1 \mathrm{H},-\mathrm{CH})$, $5.72(\mathrm{~d}, 1 \mathrm{H},-\mathrm{CH}), 6.92-6.97(\mathrm{~d}, 2 \mathrm{H}, \mathrm{Ar}-\mathrm{H}), 7.12-7.18(\mathrm{~d}, 2 \mathrm{H}, \mathrm{Ar}-\mathrm{H}), 7.54-7.57$ (t, 1H, Ar-H), 7.30-7.34 (m, 2H, Ar-H), 7.82-7.87 (m, 2H, Ar-H), 8.12 (d, 2H, Ar-H), 8.58-8.60 (d, 2H, Ar-H). ${ }^{13} \mathrm{C}$ NMR (DMSO- $d_{6}$ ): $\delta$ ppm 14.4, 44.7, 48.8, 56.9, 62.4, 66.4, 68.2, 70.2, 110.9, 120.7, 121.2, $121.7,122.0,122.5,124.3,125.8,128.4,129.6,130.6,133.7,134.9,135.7,136.6,138.3,138.5$, 
139.1, 139.7, 141.7, 147.5, 149.3, 159.8, 164.7, 169.2. MS (m/z):742 (M+1) ${ }^{+,} 743(\mathrm{M}+2)$; Anal. Calcd. for $\mathrm{C}_{38} \mathrm{H}_{32} \mathrm{ClN}_{3} \mathrm{O}_{9} \mathrm{~S}: \mathrm{C}, 61.49 ; \mathrm{H}, 4.35 ; \mathrm{N}, 5.66$; Found: C, 61.47; H, 4.31; N, 5.63\%.

4-(3-Chloro-2-(4-methoxyphenyl)-4-oxoazetidin-1-yl)-2'-(1-(3-ethoxy-4methoxyphenyl)-2-(methylsulfon-yl) ethyl)-2,4'-biisoindoline-1.1'3,3'-tetraone (6f) Yield: $68 \%$; M.P. $210-211{ }^{\circ} \mathrm{C}$; FT-IR $\left(\mathrm{KBr}, v, \mathrm{~cm}^{-1}\right)$ : 1770, 1741, 1590, 1050; ${ }^{1} \mathrm{H}$ NMR $\left(\mathrm{DMSO}-d_{6}\right): \delta 1.32\left(\mathrm{t}, 3 \mathrm{H},-\mathrm{CH}_{3}\right), 2.86\left(\mathrm{~s}, 3 \mathrm{H},-\mathrm{CH}_{3}\right), 3.42(\mathrm{t}, 1 \mathrm{H},-\mathrm{CH}), 3.79\left(\mathrm{~s}, 3 \mathrm{H},-\mathrm{OCH}_{3}\right)$, $3.88\left(\mathrm{~s}, 3 \mathrm{H},-\mathrm{OCH}_{3}\right), 4.10-4.12\left(\mathrm{q}, 2 \mathrm{H},-\mathrm{CH}_{2}\right), 4.24\left(\mathrm{~d}, 1 \mathrm{H},-\mathrm{CH}_{2}\right), 4.50(\mathrm{t}, 1 \mathrm{H},-\mathrm{N}-\mathrm{CH}), 5.21$ (d, 1H,-CH), $5.72(\mathrm{~d}, 1 \mathrm{H},-\mathrm{CH}), 6.60(\mathrm{~d}, 1 \mathrm{H}, \mathrm{Ar}-\mathrm{H}), 7.12-7.17$ (d, 2H, Ar-H), 7.20-7.25 (m, 2H, Ar-H), 7.32-7.35 (dd, 2H, Ar-H), 7.46-7.52 (m, 2H, Ar-H), 7.78-7.82 (dd, 2H, Ar-H),

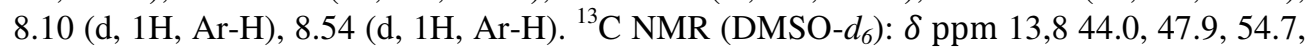
56.0, 62.4, 65.7, 67.2, 70.2, 110.4, 115.7, 120.9, 121.2, 122.8, 123.7, 125.0, 127.2, 128.4, $129.5,130.0,133.7,134.9,135.3,136.2,137.5,138.1,139.5,145.7,148.9,151.2,155.6$, 159.5, 164.9, 169.7. MS (m/z): $758(\mathrm{M}+1), 759(\mathrm{M}+2)$; Anal Calcd for $\mathrm{C}_{38} \mathrm{H}_{32} \mathrm{ClN}_{3} \mathrm{O}_{10} \mathrm{~S}: \mathrm{C}$, 60.20; H, 4.25; N, 5.54; Found: C, 60.18; H, 4.21; N, 5.49\%.

\section{Results and Discussion}

Compounds 4-amino-2'-(1-(3-ethoxy-4-methoxyphenyl)-2-(methylsulfonyl)ethyl)-2,4'-biisoindoline-1, 1', 3, 3'-tetraone 3 was prepared by condensation of 3-aminophthalic acid 2 with 1,3ethoxy-4-methoxyphenyl)-2-methylsutfonylethylamine $\mathbf{1}$ in the presence of triethylamine and acetic acid in acetonotrile solvent. The reaction was shown in Scheme 1.<smiles>CCOc1cc(C(N)CS(C)(=O)=O)ccc1OC</smiles>

Scheme 1

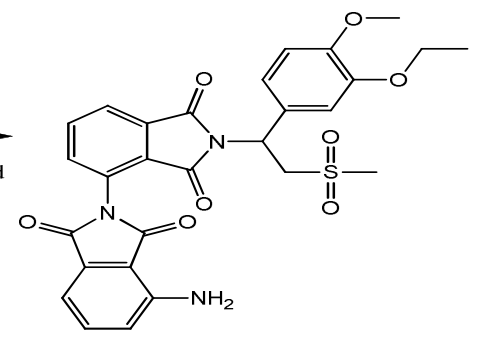

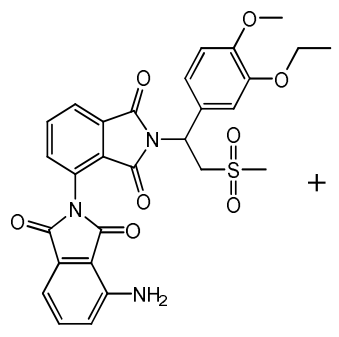

3<smiles>O=Cc1ccccc1</smiles>

$4(\mathbf{a}-\mathbf{f})$

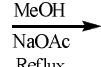

Reflux

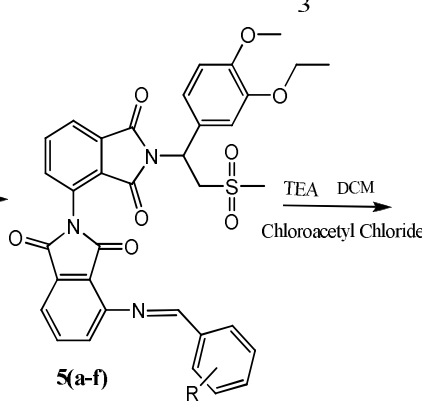<smiles>Cc1cccc([N+](=O)[O-])c1</smiles>

$\mathrm{c}$<smiles>Cc1cccc(Cl)c1Cl</smiles>

d

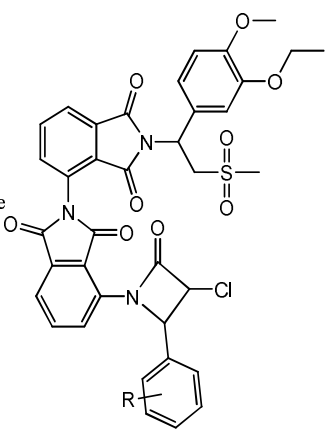

6(a-f)
$\mathrm{R}=$<smiles>Cc1ccccc1</smiles>

a<smiles>Cc1ccccc1O</smiles>

b

Scheme 2

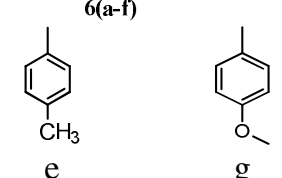

g 
Condensation of compound 3 with various aromatic aldehydes 4(a-f) give corresponding substituted Schiff base of 2'-(1-(3-ethoxy-4-methoxyphenyl)-2-(methylsulfonyl)ethyl)-2,4'biisoindoline-1,1', 3, 3'-tetra -ones 5(a-f) which upon dehydrative annulation in the presence of chloroacetylchloride and triethylamine in dichloromethane solvent yielded 4-(3-chloro-2-oxo-4arylazetidin-1-yl)-2'-(1-(3-ethoxy-4-methoxyphenyl)-2-(methylsulfonyl)-ethyl)-2,4'-iisoindoline$1.1^{\prime} 3,3^{\prime}$-tetraones 6(a-f). These reactions are summarized in Scheme 2. All the newly synthesized compounds $\mathbf{5}(\mathbf{a}-\mathbf{f})$ and $\mathbf{6}(\mathbf{a}-\mathbf{f})$ were characterized on the basis of their $\mathrm{C}, \mathrm{H}, \mathrm{N}$ analyses, Infra Red Vibrtaional, Proton and Carbon (isotope of carbon 13) NMR and mass spectral studies.

\section{Conclusion}

In this work, we have reported the synthesis of substituted Schiff base of 2'-(1-(3-ethoxy-4methoxyphenyl)-2-(methylsulfonyl)ethyl)-2,4'-biisoindoline-1,1',3,3'-tetraones and azetidinones starting from aprimilast dimer which is obtained from 3-aminophthalic acid. All the newly synthesized compounds were confirmed on the basis of their elemental analyses, IR, ${ }^{1} \mathrm{H}$ $\mathrm{NMR},{ }^{13} \mathrm{C}$ NMR and mass spectral data.

\section{Acknowledgement}

The authors are thankful to Chairman and Principal Chaitanya Post Gradauate College (Autonomous) for providing research facilities.

\section{References}

1 Sykes R B, Cimarusti C M, Bonner D P, Bush K, Floyd D M, Koster W H, Georgopapadakou N H and Liu W C, Nature, 1981, 291(5815), 489-491.

2 Kaura A, Sharama L and Dhar V J, Int J Chem Sci., 2011, 9(4), 2009-2015.

3 Rajasekharan A, Periasamy M and Venkatesan S, J Dev Biol Tissue Eng., 2010, 2(1), 5-13.

4 Basavaraj M D, Hipparagi S M and Munishama Gowda I J P T, 2011, 3(4), 3792-3801.

5 Bhat I K, Chaitanya S K, Satyanarayan P D and Kalluraya B, J Serb Chem Soc., 2007, 2(5), 437-442; DOI:10.2298/JSC0705437B

6 Chavan A A and Pai N R, Molecules, 2007, 12(11), 2467-2477;

DOI: $10.3390 / 12112467$

7 Mathew B, Mathew G E, Vijayabaskaran M and Mathew N, Der Pharma Chemica., 2010, 2(6), 238-242.

8 Ramalakshmi N, Vijayakumar R, Ilango K, Arunkumar S and Puratchikody A, Int J Chem Sci., 2008, 6(3), 1213-1222.

9 Samadhiya P, Sharma R, Srivastava S and Srivastava S K, J Sci I R Iran, 2012, 23(2), 139-146.

10 Kamble R R, Taj T, Gireesh T and Badami B V, J Chem Sci., 2011, 123(5), 657-666.

11 Vibhute Y, Chavan S, Zangade S and Vibhute A, Eur J Chem., 2013, 4(2), 98-101; DOI:10.1002/chem.201303688 